\title{
Signal and noise in ultrasound- modulated optical tomography: a Monte Carlo study
}

Gang Yao, Lihong V. Wang

Gang Yao, Lihong V. Wang, "Signal and noise in ultrasound-modulated optical tomography: a Monte Carlo study," Proc. SPIE 5320, Photons Plus Ultrasound: Imaging and Sensing, (12 July 2004); doi: 10.1117/12.532847

SPIE. Event: Biomedical Optics 2004, 2004, San Jose, CA, United States 


\title{
Signal and noise in ultrasound-modulated optical tomography: a Monte Carlo Study
}

\author{
Gang Yao ${ }^{a}$, Lihong V. Wang ${ }^{\mathrm{b}}$ \\ ${ }^{a}$ Dept of Biological Eng., University of Missouri-Columbia, Columbia, MO 65211 \\ ${ }^{b}$ Opt. Imag. Lab, Dept. of Biomed. Eng., Texas A\&M Univ., College Station, TX 77843-3120
}

\begin{abstract}
Ultrasound-modulated optical tomography in inhomogeneous scattering media was studied using a Monte Carlo modeling technique. The contributions from two different modulation mechanisms were included in the simulation. The differences between embedded absorption and scattering objects in the ultrasoundmodulated optical signals were compared. The effects of neighboring inhomogeneity and background optical properties on the ultrasound-modulated optical signals were also studied. We analyzed the signal-to-noise ratio in the experiment and found that the major noise source is the speckle noise caused by small particle movement within the biological tissue sample. This effect was studied by incorporating a Brownian motion factor in the simulation.
\end{abstract}

Keywords: Ultrasound modulated optical tomography, Monte-Carlo, Contrast, Noise

\section{INTRODUCTION}

There is a great interest in recent years to combine optical techniques with ultrasonic techniques for biomedical imaging. Various approaches have been explored, which include opto-acoustic imaging, ${ }^{1-3}$ sonoluminescence tomography, ${ }^{4}$ and ultrasound-modulated optical tomography. ${ }^{5-12}$ The advantage of these hybrid methods is that ultrasonic waves can furnish good localization information because they are much less scattering in biological tissues. In ultrasound-modulated optical tomography, some light is modulated by an ultrasonic wave inside the biological tissue to carry ultrasonic frequency. Signal processing techniques are applied to separate such tagged photons from the background un-modulated photons. The signal originations are directly related to the position of the ultrasonic column inside the tissue.

Marks et $a^{\beta}$ first investigated the possibility of probing breast cancer using this technique. Wang et $a^{6,7}$ developed ultrasound-modulated optical tomography and obtained images in tissue-simulating phantoms. Since then, many new technologies, such as parallel speckle detection by Leveque et $a^{\rho}$, frequency-swept ultrasound-modulated by Wang et $\mathrm{al}^{11}$, speckle-contrast detection by $\mathrm{Li}$ et $\mathrm{al}^{13}$, and others ${ }^{14,15}$ have been developed. In order to understand the modulation mechanisms, Wang ${ }^{16}$ developed a theoretical model that considered two important phase modulation mechanisms: the refractive index modulation and particle displacements. The analytical results agreed very well with a Monte-Carlo simulation ${ }^{17}$ for ultrasound modulation of a volumetric homogeneous scattering medium. Sakadzic and Wang ${ }^{18}$ further extended the model to include anisotropic scattering media.

For imaging applications, focusing ultrasonic transducers are often used to achieve spatial resolution. In addition, the inhomogeneous nature of turbid media adds complexity to the signal interpretation in ultrasound-modulated optical tomography. These effects cannot be studied by the analytical models used for volumetric modulation ${ }^{16-18}$. In this study, a Monte Carlo technique was used to simulate the sensitivity and the contrast of this technique. The two modulation mechanisms, i.e. refractive index modulation and particle displacement, were incorporated in the simulation. We also studied the effects of speckle noise cause by Brownian motion, which is the most important noise in experiments involving biological tissue. 


\section{METHOD}

The detected light in ultrasound-modulated optical tomography consists of two parts-the AC photons that are modulated by the ultrasonic wave and the DC background photons that are not modulated. Only those photons passing through the ultrasonic column can be modulated. The modulate depth (AC/DC) reflects the local optical and ultrasonic properties within the ultrasonic beam and can be used for tomographic imaging of the scattering medium.

In general, there are two categories of modulation processes involved: coherent modulation and noncoherent modulation. The incoherent modulation is based on the modulation of the optical properties (absorption coefficient \& scattering coefficient) of the scattering media. While the ultrasonic wave propagates in the medium, the density of the medium is changed periodically. The density change induces the change in the optical properties, which affects the output light intensity. In other words, this is an intensity modulation and does not require a coherent light source. On the other hand, the coherent modulation process is based on the modulation of phase of the scattered light. There are two different modulation mechanisms under this category. The first mechanism is due to the modulation of optical refractive index in the ultrasonic field. And the second mechanism is caused by periodic displacement of scatterers in the ultrasonic field. The coherent modulation process changes the optical pathlength of the scattered photons and eventually modulates the speckle fields at the output plane. Studies have indicated that coherent modulation is the dominant mechanism involved. In the simulation, two coherent modulation mechanisms are considered in the model.

The intensity of detected light can be written as (Wiener-Khinchin theorem):

$$
I_{n}=\frac{1}{T_{a}} \int_{0}^{T_{a}} \cos \left(n \omega_{a} \tau\right) G_{1}(\tau) d \tau
$$

where $T_{a}$ is the period of ultrasonic oscillation, $\omega_{a}$ is the ultrasonic frequency; and $G_{1}(\tau)$ is the autocorrelation function of the scattered light:

$$
G_{1}(\tau)=\int_{0}^{\infty} p(s)\left\langle E_{s}(t) E_{s}(t+\tau)\right\rangle_{t} d s,
$$

where $p(s)$ is the probability density function of pathlength $s . E_{s}$ is the electrical field of the light scattered along path s. The correlation function is calculated from the contributions from random Brownian motion (B) and ultrasonic modulation $(\mathrm{U}):^{19}$

$$
\left\langle E_{s}(t) E_{s}(t+\tau)\right\rangle=\left\langle E_{s}(t) E_{s}(t+\tau)\right\rangle_{B}\left\langle E_{s}(t) E_{s}(t+\tau)\right\rangle_{U} .
$$

The contribution from Brownian motion is determined by the particle relaxation time $\tau_{0}$, the mean free path $l$, and the total pathlength $s$ :

$$
\left\langle E_{s}(t) E_{s}(t+\tau)\right\rangle_{B}=\exp \left(-\frac{2 s}{\tau_{0} l} \tau\right) .
$$

The contribution from ultrasonic modulation can be calculated by accumulate the optical phase variations induced by ultrasound:

$$
\left\langle E_{s}(t) E_{s}(t+\tau)\right\rangle_{U}=\left\langle\exp \left[-i\left(\Delta \Phi_{n}+\Delta \Phi_{d}\right)\right]\right\rangle,
$$

where $\Delta \Phi_{. v}$ is the total phase variation caused by refractive index modulation of the scattering medium; and $\Delta \Phi_{\mathrm{d}}$ is the total phase variation caused by displacement of the scattering particles. The equations to calculate these phase variations have been given in details by Wang ${ }^{16,17}$. The modulation depth at ultrasonic frequency can be calculated as $M=l_{1} / I_{0}$ from Eq. 1 .

A Monte Carlo method was used to simulate light transport in scattering media. The basic simulation procedures have been described in detail elsewhere. ${ }^{17}$ The program was extended to handle multiple objects in order to simulate the effects of a heterogeneous background. In the simulation, the tissue sample was 
modeled as a slab containing embedded objects. For simplicity, the object and the ultrasonic column were modeled as cylinders with certain heights. The photon packet was launched perpendicularly into the tissue. Geometrical calculations were performed to determine if the path of a photon packet crossed one of the objects. The scattering angles of a photon packet were sampled using optical properties. The phase variation of a photon packet was accumulated whenever the photon intersected with the ultrasound column or was scattered within the ultrasound column. The correlation function of the transmitted light was calculated by accumulating contributions from all transmitted photons.

Unless indicated specifically, the following optical properties of the background scattering media were used in the simulation: ${ }^{20}$ the refractive index $n=1.33$, the absorption coefficient $\mu_{\mathrm{a}}=0.1 \mathrm{~cm}^{-1}$, the scattering coefficient $\mu_{\mathrm{s}}=20.0 \mathrm{~cm}^{1}$, and the anisotropy factor $g=0.9$. The thickness of the tissue slab is $3 \mathrm{~cm}$. Both the object and ultrasonic column have a radius of $1 \mathrm{~mm}$ and a height of $10 \mathrm{~mm}$ although their dimensions are not required to be identical in the simulations. The velocity of the ultrasonic wave is $1480 \mathrm{~m} / \mathrm{s}$. The ultrasound amplitude is $0.1 \mathrm{~nm}$. The ultrasound wave vector is $4 \times 10^{3} \mathrm{~m}^{-1}$ that corresponds to a wavelength of $1.57 \mathrm{~mm}$ in the scattering media. A light beam of a radius of $1 \mathrm{~cm}$ is incident perpendicularly upon the turbid medium. Only the transmitted photons within a circular disk of a $1-\mathrm{cm}$ radius on the exit plane, simulating the detection area, were scored.

\section{RESULTS}

\subsection{Sensitivity to localized inhomogeneous optical properties}

In ultrasound-modulated optical tomography, the modulated photons carry the optical as well as ultrasonic properties of the ultrasonic column. If the optical properties within the ultrasonic column are different, the detected signal will be different. Ultrasonic heterogeneity and Brownian motion were not

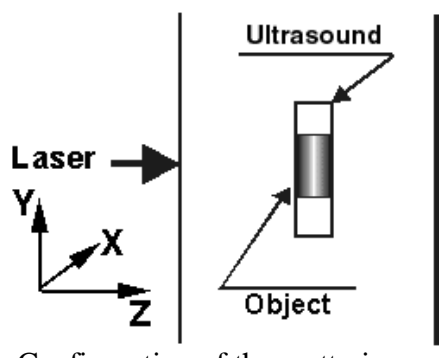

Fig.1 Configuration of the scattering medium and the ultrasound considered in this simulation. In order to study the effects of these different optical properties, we simulated objects with different absorption coefficients and scattering coefficients. The simulation configuration is shown in Fig. . A single cylindrical object is buried at the center of the tissue slab. The position of the ultrasonic column is aligned with the object. Therefore, every photon passing through the object will be labeled by the ultrasound.

Simulation results are shown in Fig.2. The values in the plots in Figs. 2(a)-2(c) are normalized to the signal values obtained when the embedded object has the same optical properties of the background medium.
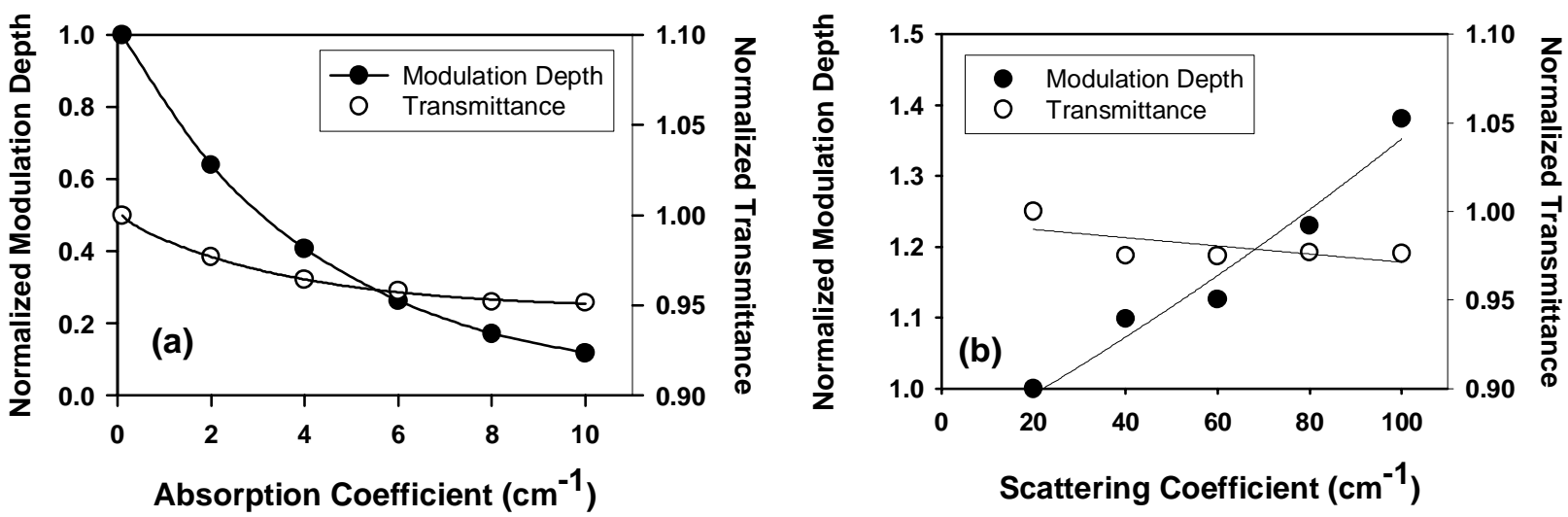


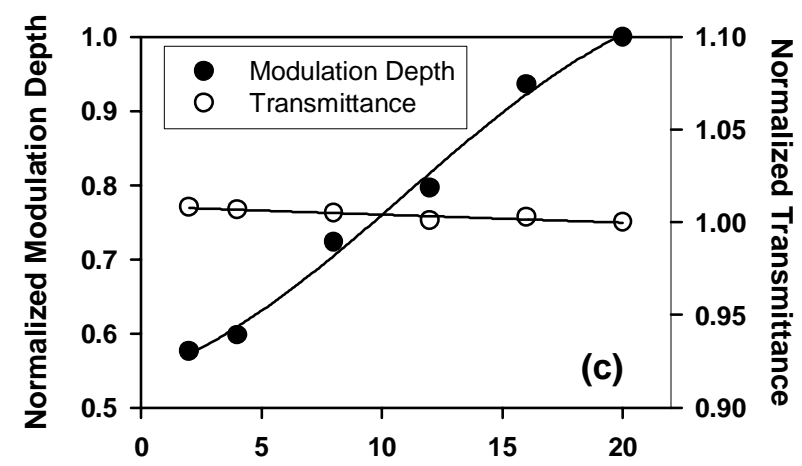

Reduced Scattering Coefficient $\left(\mathrm{cm}^{-1}\right)$
Fig. 2 Modulation depth and total transmittance versus the (a) absorption coefficient (b) scattering coefficient, and (c) reduced scattering coefficient. The values in the plots are normalized to the signal values obtained when the embedded object has the same optical properties of the background medium

From the simulation results, the modulation signal is much more sensitive to the change of optical properties than the total output intensity (transmittance). For example, as the optical absorption coefficient of the object increases 100 times, the modulation depth decreases by $~ 88 \%$, while the transmittance only decreases by $\sim 5 \%$. The modulation depth decreases as the absorption coefficient of the object increases because more photons passing through the object have been absorbed. If only the absorption coefficient $\mu_{a}$ of the object is the changed by $\Delta \mu_{a}$, the modulation signal changes by

$$
\Delta M \propto-\int M(p) \exp \left(-\Delta \mu_{a} p\right) f(p) d p,
$$

where $\mathrm{M}(p)$ is the intensity of modulated photons having path length $p$ inside the object before the absorption coefficient is varied; and $f(p)$ is the distribution of $p$. When the absorption coefficient is changed by 100 fold from $0.1 \mathrm{~cm}^{-1}$ to $10 \mathrm{~cm}^{-1}$, the modulated photons of $p$ values that is comparable with the $0.2-\mathrm{cm}$ diameter of the embedded object will vary by a factor of $\exp (-9.9 \times 0.2)=14 \%$, which roughly matches the simulated $\sim 88 \%$ decrease.

The scattering properties ( $\mu_{\mathrm{s}}$ and $g$ ) of the embedded object have even less effect on the total transmittance than the absorption property $\left(\mu_{\mathrm{a}}\right)$ has. However, the modulation depth still shows significant sensitivities for these changes. In the example, when the scattering increases from $20 \mathrm{~cm}^{-1}$ to $100 \mathrm{~cm}^{-1}$, the modulation depth increases by $\sim 40 \%$; while the total transmission only decreases by less than $3 \%$. If the scattering properties of the object are changed, the paths of photons will be altered. Although a simple expression similar to Eq. (6) does not exist, the increase of the scattering coefficient of the embedded object causes the photons to have more scattering events and longer pathlength within the ultrasound column. Both effects will lead to a higher modulation depth. Fig. 2(c) shows that the modulation depth decreases as the anisotropic factor of the embedded object increases. In Fig.2(c), the scattering coefficient of the object was fixed at 20 $\mathrm{cm}^{-1}$ in the simulation. Calculation indicated that the average pathlength inside ultrasound column is higher at smaller anisotropic factor, which leads to higher modulation depth because of the refractive index modulation mechanism.

\subsection{Effect of background optical properties}

The signal level is affected by the optical properties of the background medium. In Fig. 3(a), the modulation signal of a single object was calculated with different background scattering coefficients. The object was positioned at the center of the turbid medium. Its optical properties are: $\mu_{\mathrm{a}}=2 \mathrm{~cm}^{-1}, \mu_{\mathrm{s}}=100.0 \mathrm{~cm}^{1}$, and $\mathrm{g}=$ 0.9. It is interesting to see that modulation depth increases as the background scattering coefficient increases because more photons interact with the ultrasonic column. Both modulation mechanisms contribute to this effect. Detailed calculations show that both the average scattering events of the modulated photons and their pathlenths within the ultrasound column increase as the background scattering coefficient increases, which explains the above observation. Fig. 3(b) shows the effect of background absorption coefficient. The object's optical properties are: $\mu_{\mathrm{a}}=2 \mathrm{~cm}^{-1}, \mu_{\mathrm{s}}=20.0 \mathrm{~cm}^{1}$, and $g=0.9$. The modulation depth increases slightly as the background absorption coefficient increases because more unmodulated photons are absorbed. The increase is mainly due to the modulation of refractive index. 


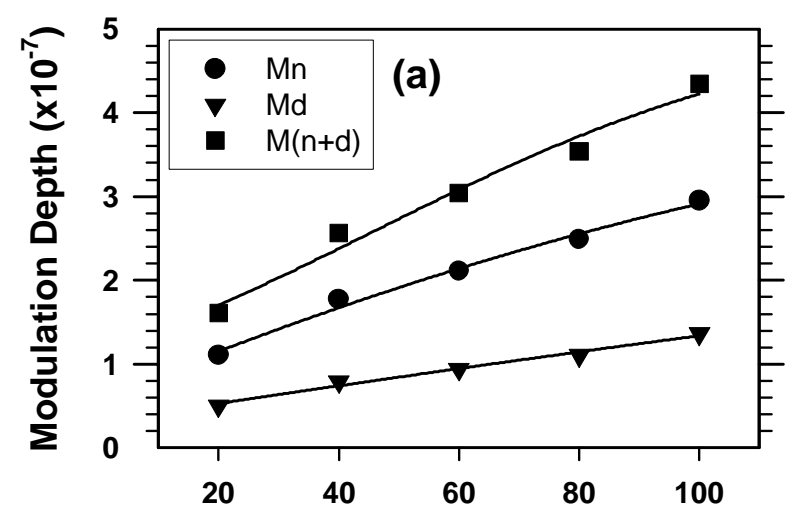

Scattering Coefficient $\left(\mathrm{cm}^{-1}\right)$

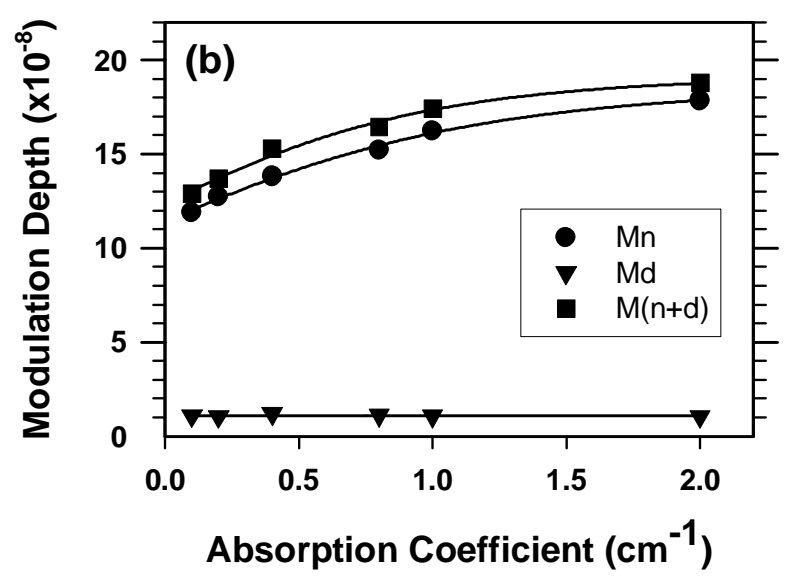

Fig.3. Modulation depth and total transmittance versus the background (a) scattering coefficient and (b) absorption coefficient. $M(n)$ : Refractive index modulation; $M(d)$ : particle displacement modulation; $M(n+d)$ : Modulation by both mechanisms.

\subsection{Effect of inhomogeneity}

In ultrasound-modulated optical tomography, two-dimensional images are usually obtained by mechanically scanning the ultrasonic transducer over the sample. A measured modulation depth is directly assigned as signal intensity at the corresponding ultrasound location. Because the local modulated signal intensity depends on the local optical fluence, the internal optical fluence affects the obtained images. Because the light fluence distribution is not uniform within the scattering medium, the modulation depth is related to the position of the ultrasonic column even in a homogeneous medium. ${ }^{10}$

In reality, tissue samples are inhomogeneous in both absorption and scattering properties. A buried object will affect the optical fluence in its neighborhood and the modulated signal intensity. Figure 4 shows such an example in which two identical objects are separated by $3 \mathrm{~mm}$ along the $z$-axis. The ultrasonic column is located at Object-1, which is at the center of the slab. Brownian motion is not considered in the simulation. From the results [Figs. 4(a) \& 4(b)], the calculated modulation depth is affected by the optical properties of the other neighboring object. As the absorption coefficient and the scattering coefficient of Object- 2 increase, the signal values of Object- 1 decrease because the modulated light decreases after passing through Object2. In other words, Object-2 casts a shadow on Object-1 when an image of the scattering medium is produced. Such "cross talks" depends on the optical properties, the distant, and the size of neighboring objects.

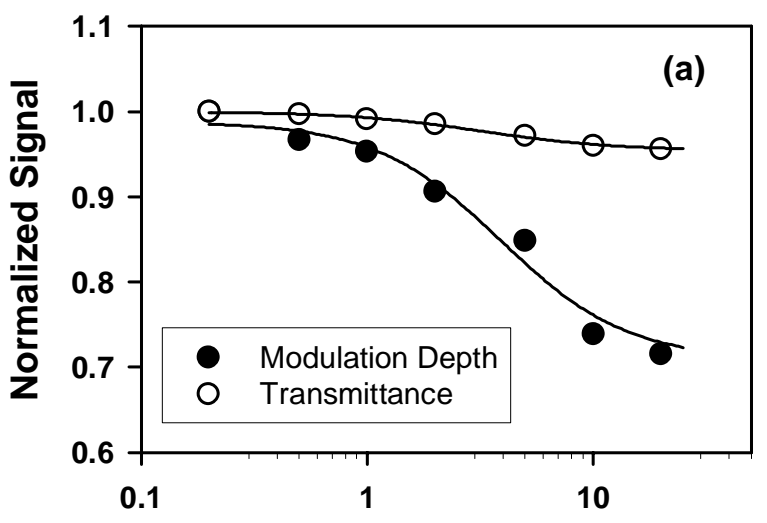

Absorption Coefficient $\left(\mathrm{cm}^{-1}\right)$

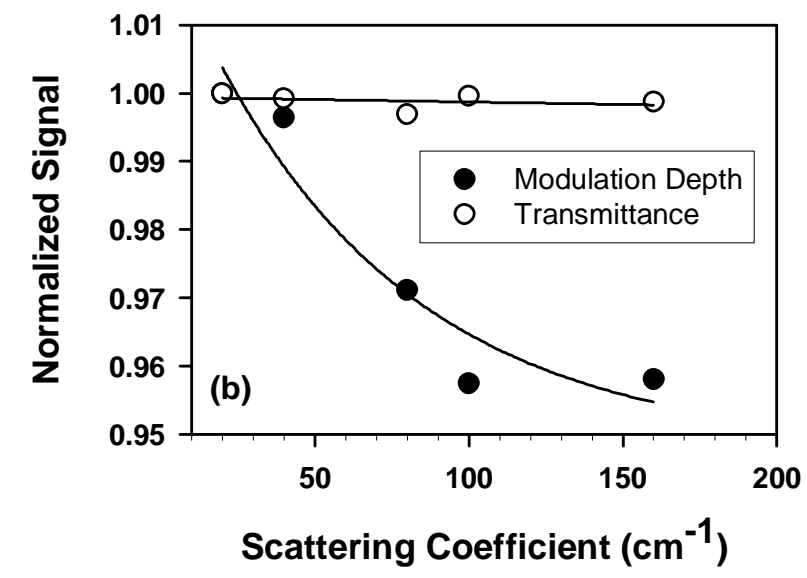

Fig.4. Modulation depth and total transmittance versus the (a) absorption coefficient and (b) scattering coefficient of the second object. The two objects are separated by $3 \mathrm{~mm}$ along the $z$-axis. 
The above several examples indicate that the signal intensity is indeed related to the background properties and object distribution in the scattering medium. Therefore, quantitative measurements of the optical properties of the embedded objects entail sophisticated reconstruction algorithms.

\subsection{Noise and speckle}

In ultrasound-modulated optical tomography, the signal-to-noise ratio (SNR) is very critical because the modulated signal is very small. In order to improve experimental results, system noise properties should be carefully analyzed and optimized. There are many possible noise sources in the experimental system including contributions from both the optical system and ultrasonic system. Among all the noise sources, the shot noise is the theoretical limit of noise and the speckle noise appears to be the most significant noise source in biological tissue. Speckle noise is caused by the random movement of small particles within the biological tissue. These particle movements change the speckle patterns and induce noise in the detected optical signals.

The speckle correlation time is related to the particle relaxation time in the scattering medium. With a small relaxation time, particles move fast and cause short speckle correlation time. The effect of random movement of small particles was simulated by incorporating Brownian motion (Eq. 4) into the Monte Carlo simulation. Photons that are not interacting with ultrasound are dominant in this effect because their amount is much larger than modulated photons. Figure 5 shows an example of signal sensitivity dependency on the particle relaxation time. Two small absorption objects were used in the simulation. Their absorption coefficients are $0.2 \mathrm{~cm}^{-1}$ and $2.0 \mathrm{~cm}^{-1}$ respectively. The Y-axis in Fig. 5 is plotted as relative modulation depth that is defined as $\left[\mathrm{M}\left(\mu_{\mathrm{a}}=2.0\right)-\mathrm{M}\left(\mu_{\mathrm{a}}=0.2\right)\right] / \mathrm{M}\left(\mu_{\mathrm{a}}=0.2\right)$. At small particle relaxation time, the modulation depths obtained at these two objects are indistinguishable. The signal difference becomes larger as the relaxation time increases. The results also show that modulation depth at a higher ultrasonic frequency has better sensitivity than that at a lower ultrasonic frequency. This is because the autocorrelation signal is calculated within one ultrasonic period which is smaller at high frequency. This result implicate that the speckle decorrelation problem may have less effect when high frequency ultrasound is applied.

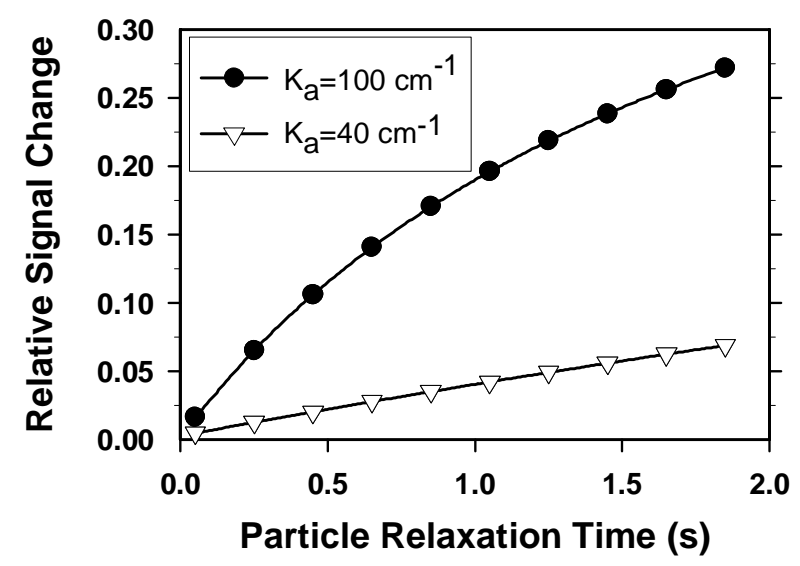

Fig.5 Effect of particle relaxation time on signal sensitivity.

\section{CONCLUSIONS}

A Monte Carlo simulation was applied to study the signal sensitivity of ultrasound-modulated optical tomography. The simulation study indicates that this technology is significantly more sensitive to small optical objects than unmodulated intensity measurements. In addition, an inhomogeneous tissue background will 
alter the signal intensity. Therefore, obtaining accurate optical properties by simple direct imaging scanning requires the use of sophisticated reconstruction.

Optical speckles play an extremely important role in the experiments. Time variation of speckle patterns represents the largest source of noise in an experiment, especially if the biological tissue is thick. To overcome this problem, data must be acquired within the speckle correlation time. Our study also indicated that ultrasound modulation with higher frequency has better immunization to speckle variations.

\section{Acknowledgments}

This project was sponsored by a Summer Faculty Resaerch Fellowship support (for Yao Gang) from University of Missouri-Columbia; and in part (for Wang) by National Institutes of Health grants R29 CA68562 and R01 CA71980, National Science Foundation grant BES-9734491, and Texas Higher Education Coordinating Board grant 000512-0063-2001.

\section{REFERENCES}

1. R. A. Kruger, P. Liu, "Photoacoustic ultrasound: Theory," in Laser-Tissue Interaction V, S. L. Jacques, ed., Proc. SPIE 2134A, 114-118 (1994).

2. A. A. Oraevsky, R. O. Esenaliev, S. L. Jacques, F. K. Tittel, "Laser optic-acoustic tomography for medical diagnostics: principles," in Biomedical Sensing, Imaging, and Tracking Technologies I, R. A. Lieberman, H. Podbielska, and T. Vo-Dinh, eds., Proc. SPIE 2676, 22-31 (1996).

3. C. G. A. Hoelen, F. F. M. De Mul, R. Pongers, and A. Dekker, "Three-dimentional photoacoustic imaging of blood vessels in tissue," Opt. Lett. 23, 648-650 (1998).

4. L.-H. Wang, and Q. Shen, "Sonoluminescence tomography of turbid media," Opt. Lett. 23, 561-563 (1998).

5. F. A. Marks, H. W. Tomlinson, and G. W. Brooksby, "Comprehensive approach to breast cancer detection using light: photon localization by ultrasound modulation and tissue characterization by spectral discrimination," in Photon Migration and Imaging in Random Media and Tissue, B. Chance and R. R. Alfano, eds., Proc. SPIE 1888, 500-510 (1993).

6. L.-H. Wang, S. L. Jacques, and X. Zhao, "Continues-wave ultrasonic modulation of scattered laser light to image objects in turbid media," Opt. Lett. 20, 629-631 (1995).

7. L.-H. Wang and X. Zhao, "Ultrasound-modulated optical tomography of absorbing objects buried in dense tissuesimulating turbid media," Appl. Opt. 36, 7277-7282 (1997).

8. Kempe, M. Larionov, D. Zaslavsky, and A. Z. Genack, "Acousto-optic tomography with multiple scattered light," J. Opt. Soc. Am. 14, 1151-1158 (1997).

9. S. Leveque, A. C. Boccara, M. Lebec, and H. Saint-Jalmes, "Ultrasonic tagging of photon paths in scattering media: parallel speckle modulation processing,” Opt. Lett. 24, 181-183 (1999).

10. G. Yao and L.-H. Wang, "Theoretical and experimental studies of ultrasound-modulated optical tomography in biological tissue," Appl. Opt., 39, 659-664 (2000).

11. L.-H. Wang, and G. Ku, "Frequency-swept ultrasound-modulated optical tomography of scattering media," Opt. Lett. 23, 975-977 (1998).

12. G. Yao, S. Jiao, and L.-H. Wang, "Frequency-swept ultrasound-modulated optical tomography in biological tissue by use of parallel detection," Opt. Lett., 25, 734-736 (2000).

13. J. Li, S. Sakadzic, G. Ku, L.-H. Wang, "Transmission- and side-detection configurations in ultrasound-modulated optical tomography of thick biological tissues," Appl. Opt. 42 , 4088-4094(2003).

14. J. Selb, L. Pottier, A.C. Boccara, "Nonlinear effects in acousto-optic imaging," Opt. Lett. 27, 918-920(2002). 
15. A. Lev, B.G. Sfez BG, "Direct, noninvasive detection of photon density in turbid media Opt. Lett. 27, 473475(2002).

16. L.-H. Wang, "Mechanisms of ultrasonic modulation of multiply scattered coherent light: an analytic model," Physical Review Letters 8704, 3903-(1-4) (2001).

17. L.-H. Wang, "Mechanisms of ultrasonic modulation of multiply scattered coherent light: a Monte Carlo model," Optics Letters 26, 1191-1193 (2001).

18 S. Sakadzic, and L.-H. Wang, "Ultrasonic modulation of multiply scattered coherent light: An analytical model for anisotropically scattering media," Phys. Rev. E 66, 026603(2002).

19. W. Leutz, and G. Maret, "Ultrasonic modulation of multiply scattered light," Physica B 204, 14-19 (1995).

20. G. Marquez, L.-H. Wang, S.-P. Lin, J. A. Schwartz, and S. L. Thomsen, "Anisotropy in the absorption and scattering spectra of chicken breast tissue," Appl. Opt. 37, 798-805 (1998). 